\title{
miRNA-125a-5p inhibits hepatocellular carcinoma cell proliferation and induces apoptosis by targeting TP53 regulated inhibitor of apoptosis 1 and Bcl-2-like-2 protein
}

\author{
MA MING ${ }^{1}$, MA YING ${ }^{2}$ and $\mathrm{MALING}^{3}$ \\ ${ }^{1}$ Department of Hepatobiliary Surgery, People's Hospital of Xinjiang Uygur Autonomous Region, Urumqi, \\ Xinjiang 830001; ${ }^{2}$ Department of Clinical Laboratory, The First Affiliated Hospital of Xinjiang Medical University, \\ Urumqi, Xinjiang 830054; ${ }^{3}$ Department of Pharmacy, Xinjiang Uygur Autonomous Region Chest Hospital, \\ Urumqi, Xinjiang 830049, P.R. China
}

Received July 23, 2018; Accepted April 11, 2019

DOI: $10.3892 /$ etm.2019.7674

\begin{abstract}
The present study aimed to investigate the role and underlying molecular mechanism of microRNA (miR)-125a-5p in hepatocellular carcinoma. The level of miR-125a-5p was detected using reverse transcription-quantitative polymerase chain reaction. TargetScan was used to investigate the association between miR-125a-5p and TP53-regulated inhibitor of apoptosis 1 (TRIAP1)/B cell lymphoma-2-like 2 protein (BCL2L2). Dual luciferase reporter assay was used to confirm this prediction. To investigate the role of miR-125a-5p in hepatocellular carcinoma (HCC) cells, miR-125a-5p was overexpressed in the human HCC cell line PLC/PRF/5 using miR-125a-5p mimics. Subsequently, cell proliferation, cell apoptosis and cell migration were studied using MTT assay, flow cytometry analysis and Transwell assay, respectively. Protein expression levels in the present study were measured by western blot analysis. Taken together, the present results suggested that miR-125a-5p was markedly downregulated in HCC cells. TRIAP1 and BCL2L2 were direct targets of miR-125a-5p and were upregulated in PLC/PRF/5 cells. miR-125a-5p upregulation inhibited PLC/PRF/5 cell viability and migration and induced cell apoptosis. In addition, miR-125a-5p overexpression increased the expression of caspase 9 and apoptotic protease-activating factor 1 . Notably, the present study revealed that all the effects on PLC/PRF/5 cells elicited by miR-125a-5p overexpression were eliminated by TRIAP1/BCL2L2 upregulation. In conclusion, miR-125a-5p was shown to be downregulated in hepatocellular carcinoma
\end{abstract}

Correspondence to: Dr Ma Ming, Department of Hepatobiliary Surgery, People's Hospital of Xinjiang Uygur Autonomous Region, 91 Tianchi Road, Urumqi, Xinjiang 830001, P.R. China

E-mail: maming330@sohu.com

Key words: hepatocellular carcinoma, microRNA-125a-5p, TP53-regulated inhibitor of apoptosis 1, B cell lymphoma-2-like 2 protein, apoptosis, migration and its upregulation inhibited hepatocellular carcinoma cell growth and metastasis by targeting TRIAP1 and BCL2L2.

\section{Introduction}

Hepatocellular carcinoma (HCC) is the sixth most common cancer worldwide, and is associated with almost half a million cases of mortality every year (1). In early stage HCC, potential curative treatments including surgical resection, percutaneous ablation and liver transplantation are available (2). However, for advanced HCC, curative treatments are not available and the 5-year survival rate for advanced stage patients is dismal (3). Therefore, the identification of potential early biomarkers and discovery of novel therapies are urgently required, and numerous methods are undergoing development for this purpose (4).

MicroRNAs (miRNAs/miRs) are a class of endogenous, single-stranded, small non-coding RNAs that are 18-25 nucleotides in length, and function as negative regulators of gene expression (5). miRNAs are able to trigger either mRNA degradation or translational repression by binding to the 3'-untranslated region (UTR) of specific mRNAs with perfect or near-perfect complementarity, respectively (6). Several hundred miRNAs have been described in humans. The basic role of miRNAs either as oncogenes or as tumor suppressors in human HCC has been identified $(7,8)$. However, the specific role of aberrantly expressed miRNAs is yet to be elucidated. The present hypothesis was that aberrantly expressed miRNAs may contribute to tumor growth and migration by modulating the expression of gene products involved in phenotypic characteristics of cancer cells, including cell growth, migration and invasion. RNA interference analysis has previously been used in HCC treatment $(9,10)$. Detectable miRNAs associated with liver tumor tissue, serum, plasma and urine could provide an effective means to monitor response to therapies and to establish prognosis (5).

miR-125a-5p is a miRNA that has been extensively studied in tumors. In lung cancer, miRNA-125a-5p was revealed to be a tumor suppressor that directly targets the signal transducer and activator of transcription 3 and neural precursor cell expressed 
developmentally downregulated $9(11,12)$. Natalia, et al $(13)$ reported that miR-125a-5p could repress cervical cancer cell invasion by targeting mitogen activated protein kinase 1 . Cao et al (14) suggested that miRNA-125a-5p inhibited gastric cancer cell invasion and metastasis by regulating the expression of breast carcinoma metastasis suppressor gene 1 . Furthermore, miRNA-125a-5p has been shown to inhibit cell growth in hepatitis B virus-associated hepatocellular carcinoma by targeting the tyrosine protein kinase receptor, ErbB3 (15). However, to the best of our knowledge, the role and mechanism of miR-125a-5p in hepatocellular carcinoma remains largely unknown.

The purpose of the current study was to investigate the role and mechanism of miR-125a-5p in the development of hepatocellular carcinoma.

\section{Materials and methods}

Cell culture. The human hepatocellular carcinoma cell lines PLC/PRF/5 and MHCC97L, and the human immortalized liver cell line HL-7702 were purchased from the American Type Culture Collection. All cells were grown in Dulbecco's modified Eagle's medium (DMEM; Thermo Fisher Scientific, Inc.) supplemented with $10 \%$ (v/v) fetal bovine serum (FBS), and incubated in a $95 \%$ humidified incubator $\left(5 \% \mathrm{CO}_{2}, 37^{\circ} \mathrm{C}\right)$.

Cell transfection. PLC/PRF/5 cells $\left(5 \times 10^{4}\right.$ cells per well) were seeded into 6-well plates and incubated in standard conditions $\left(5 \% \mathrm{CO}_{2}, 37^{\circ} \mathrm{C}\right), 24 \mathrm{~h}$ prior to cell transfection. Subsequently, miR-125a-5p mimic (5'-UCCCUGAGACCCUUUAACCUG UGA-3'), mimics control (5'-UUCUCCGAACGUGUCACG UTT-3'), TRIAP1-plasmid, BCL2L2-plasmid, miR-125a-5p mimic + TRIAP1-plasmid or miR-125a-5p mimic + BCL2L2-plasmid (Biovector NTCC Inc.) were transfected into the PLC/PRF/5 cells using $30 \mu \mathrm{l}$ Lipofectamine $2000^{\mathrm{TM}}$ reagent (Invitrogen; Thermo Fisher Scientific, Inc.) according to the manufacturer's protocol. A total of $0.67 \mu \mathrm{g}(50 \mathrm{pmol})$ miR-125a-5p mimic or mimic control, $25 \mu 1$ TRIAP1-plasmid solution $(0.12 \mathrm{~g} / \mathrm{l})$ or BCL2L2-plasmid solution $(0.12 \mathrm{~g} / \mathrm{l})$ was used for transfection. Following incubation for $48 \mathrm{~h}$, cells were ready for subsequent experimentation. Cells without any treatment were considered as the control group (Control).

Reverse transcription-quantitative PCR (RT-qPCR). Total RNA was extracted from the cells using TRIzol ${ }^{\circledR}$ reagent (Invitrogen $^{\mathrm{TM}}$; Thermo Fisher Scientific, Inc.) according to the manufacturer's protocol. The RNAs were reverse transcribed to synthesize the cDNAs using PrimeScript RTreagent kit (Takara Biotechnology Co., Ltd.) according to the manufacturer's protocol. Subsequently, qPCR was performed to analyze the cDNAs using a TaqMan Universal PCR Master Mix kit (Thermo Fisher Scientific, Inc.). The thermocycling conditions were as follows: $95^{\circ} \mathrm{C}$ for $10 \mathrm{~min}$, followed by 38 cycles of $95^{\circ} \mathrm{C}$ for $10 \mathrm{sec}$ and $58^{\circ} \mathrm{C}$ for $60 \mathrm{sec}$. The primer sequences were as follows: U6 forward, 5'-CTCGCTTCGGCAGCA CA-3' and reverse, 5'-AACGCTTCACGAATTTGCGT-3'; GAPDH forward, 5'-GAAGGTGAAGGTCGGAGTC-3' and reverse, 5'-GAAGATGGTGATGGGATTTC-3'; miR-125a-5p forward, 5'-CGATTCCCTGAGACCCTTTAA-3' and reverse, 5'-TATGGTTTTGACGACTGTGTGAT-3'; TRIAP1 forward,

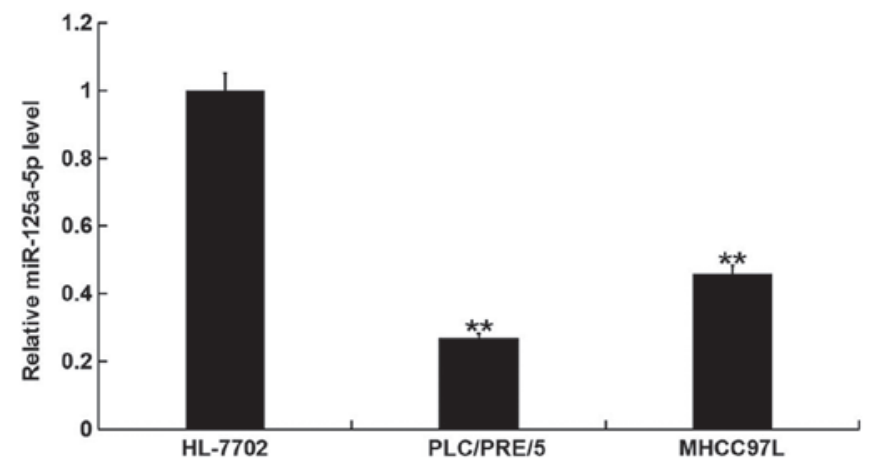

Figure 1. Detection of expression levels of miR125a-5p in PLC/PRF/5 and HL-7702 cells. Expression level of miR-125a-5p was significantly lower in the PLC/PRF/5 and the MHCC97L cells, compared with the HL-7702 cells ${ }^{* * *} \mathrm{P}<0.01$. miR, microRNA.

5'-TATCTTGCAGGAACTGTGTGCTA-3' and reverse, 5'-AATTTAGGTTCTTCCTCCACAGC-3'; BCL2L2 forward, 5'-TGAGTTCGAGACCCGCTTC-3' and reverse, 5'-AAA AGTTCATCGGAGACCTG-3'. GAPDH and U6 were used as the internal controls for mRNA and miRNA expression, respectively. The $2^{-\Delta \Delta \mathrm{Cq}}$ method (16) was used to quantify relative gene expressions.

Western blot analysis. Cells were harvested and lysed using radio immunoprecipitation assay buffer containing protease inhibitor (Abcam). The concentration of protein samples was determined with a bicinchoninic acid Protein Assay kit (Bio-Rad Laboratories, Inc.). Equal amount of proteins (30 $\mu \mathrm{g}$ per lane) were separated via SDS-PAGE on $12 \%$ gels then transferred on polyvinylidene fluoride membranes (Bio-Rad Laboratories, Inc.). After blocking with 5\% non-fat milk at room temperature for $2 \mathrm{~h}$, the membranes were blotted overnight with the primary antibodies anti-TRIAP1 (cat no. KL507313; Kanglang Biotechnology Co., Ltd.; 1:2,000) and anti-BCL2L2 (cat no. ab38629; Abcam; 1:500) at $4^{\circ} \mathrm{C}$. The next day, membranes were incubated with anti-rabbit horseradish peroxidase-linked IgG secondary antibody (cat no. 7074; Cell Signaling Technology, Inc.; 1:2,000) at room temperature for $4 \mathrm{~h}$. Finally, protein bands were visualized using the enhanced chemiluminescence detection system (Super $^{\mathrm{TM}}$ Signal West Dura Extended Duration substrate; Thermo Fisher Scientific, Inc.) with the intensity analyzed with Image J software (version 1.8.0; National Institutes of Health) with GAPDH as the loading control.

MTT assay. MTT assay was used to assess cell viability. Briefly, PLC/PRF/5 cells were harvested and then reseeded into 96 -well culture plates and cultured at $37^{\circ} \mathrm{C}$ for 24,48 or $72 \mathrm{~h}$. Subsequently, MTT solution (in thiazolyl blue tetrazolium bromide; Amresco LLC) was added into each culture well and incubated for a further $4 \mathrm{~h}$. Finally, the optical density values were measured at $570 \mathrm{~nm}$ using a Synergy ${ }^{\mathrm{TM}}-2$ Multi-function microplate reader (Bio Tek Instruments, Inc.).

Cell apoptosis assay. Following $48 \mathrm{~h}$ of incubation, $3 \times 10^{3}$ cells/well were seeded into 96-well plates and labeled with Annexin V-fluorescein isothiocyanate (FITC) and propidium 


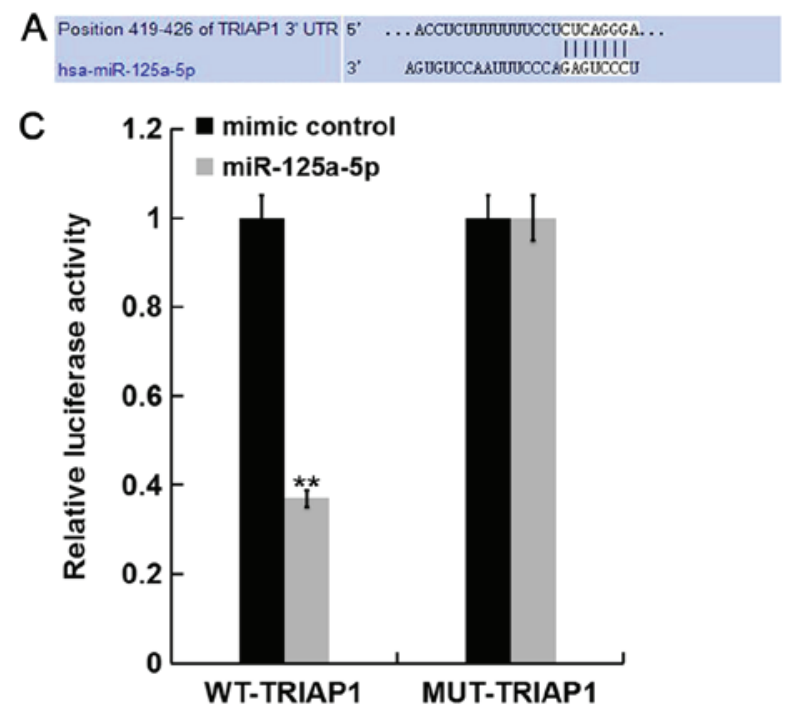

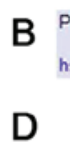

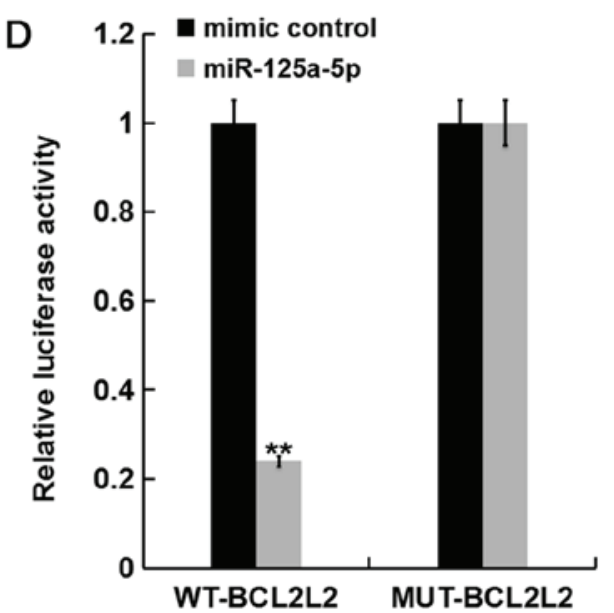

Figure 2. Detection of potential targets of miR-125a-5p. (A) Alignment of one predicted miR-125a-5p target site in the TRIAP1-3'-UTR. (B) Alignment of one predicted miR-125a-5p target site in the BCL2L2-3'-UTR. (C) Predicted miR-125a-5p binding sites (seed sequence) of the wild-type and mutated TRIAP1 3'-UTR are shown. (D) Predicted miR-125a-5p binding sites (seed sequence) of the wild-type and mutated BCL2L2-3'-UTR compared with the mimics control, ${ }^{* *} \mathrm{P}<0.01$ vs. mimics control group. BCL2L2, B-cell lymphoma-2-like 2 protein; miR, microRNA; MUT, mutant; TRIAP1, TP53 regulated inhibitor of apoptosis 1; 3'-UTR, 3'-untranslated region; WT, wild type.

iodide (PI; Cell Signaling Technology, Inc.), as per as the manufacturer's protocol. The BD LSRFortessa X-20 flow cytometer (BD Biosciences) with FlowJo software (version 10.0.6; BD Biosciences) was used to analyze cell apoptosis.

Cell migration assay. The transfected PLC/PRF/5 cells $\left(1 \times 10^{5}\right)$ in serum-free medium were placed into the upper chamber of a 24-well plate with $8 \mathrm{~mm}$ diameter (Corning, Incorporated). A total of $500 \mu \mathrm{l}$ DMEM containing 10\% FBS was added in the bottom chamber, and the cells were subsequently incubated at $37^{\circ} \mathrm{C}$ for $48 \mathrm{~h}$. The cells in the upper chamber were removed using a cotton swab, and the membranes were stained with $0.1 \%$ crystal violet for $20 \mathrm{~min}$ at room temperature. Finally, the cells were counted in 10 random fields under a light microscope (magnification, $\mathrm{x} 200$ ).

Dual-luciferase reporter assay. TargetScan bioinformatics software (www.targetscan.org) was used to predict the potential targets of miR-125a-5p, the findings revealed that TRIAP1 and BCL2L2 were potential targets of miR-125a-5p. To confirm this prediction, $\mathrm{PLC} / \mathrm{PRF} / 5$ cells $\left(5 \times 10^{4}\right.$ cells) were seeded into each well of a 24 -well plate. After $24 \mathrm{~h}$, the cells were co-transfected with TRIAP1/BCL2L2 3'-UTRpmirGLO plasmid (BioVector NTCC Inc), containing mutant TRIAP1/BCL2L2 3'-UTR or wild type TRIAP1/BCL2L2 3'-UTR, and miR-125a-5p mimic or mimic control using Lipofectamine $^{\circledR} 2000$ reagent (Invitrogen; Thermo Fisher Scientific, Inc.), according to the manufacturer's protocol. Following incubation at $37^{\circ} \mathrm{C}$ for another $48 \mathrm{~h}$, the luciferase activity (Luciferase Reporter Assay Kit; AmyJet Scientific, Inc.) was assessed using the dual-luciferase reporter assay system (Promega Corporation) and Renilla luciferase was used as the internal control for the normalization of results.

Statistical analysis. All experiments were repeated at least three times. Data are presented as the mean \pm standard deviation. Statistical significance was assessed using SPSS 19.0 statistical software (IBM Corp.). Differences between multiple groups were assessed by one way analysis of variance followed by Tukey's post hoc test and differences between two groups were analyzed with Student's t-test. $\mathrm{P}<0.05$ was considered to indicate a statistically significant difference.

\section{Results}

miR-125a-5p is downregulated in human hepatocellular carcinoma cells. The expression of miR-125a-5p was detected in the human hepatocellular carcinoma cell line, PLC/PRF/5, and the human immortalized liver cell line, HL-7702, using RT-qPCR. The results indicated that, compared with the HL-7702 cells, the level of miR-125a-5p was markedly downregulated in PLC/PRF/5 cells and MHCC97L cells. The decrease of miR-125a-5p expression in PLC/PRF/5 cells was greater than in MHCC97L cells (Fig. 1), therefore, PLC/PRF/5 cells were selected for subsequent experimentation.

miR-125a-5p directly targets TRIAP1 and BCL2L2. TargetScan database was used to predict the potential targets of miR-125a-5p. TRIAP1 and BCL2L2 were revealed to be the predicted targets of miR-125a-5p (Fig. 2A and B). To investigate whether TRIAP1 and BCL2L2 are direct targets of miR-125a-5p, luciferase reporter assay was performed. The results indicated that miR-125a-5p markedly reduced the luciferase activity of TRIAP1-3'-UTR and BCL2L2-3'-UTR in PLC/PRF/5 cells, but exerted no effect on the mutant form of TRIAP1-3'-UTR and BCL2L2-3'-UTR (Fig. 2C and D), indicating that TRIAP1 and BCL2L2 are direct targets of miR-125a-5p in PLC/PRF/5 cells.

TRIAPI and BCL2L2 are upregulated in human hepatocellular carcinoma cells. The human hepatocellular carcinoma cell line, PLC/PRF/5 and the human immortalized liver cell 
A

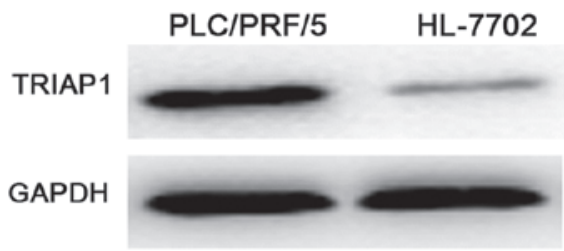

京

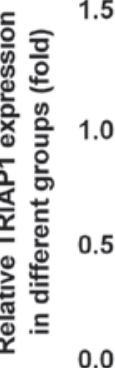

B
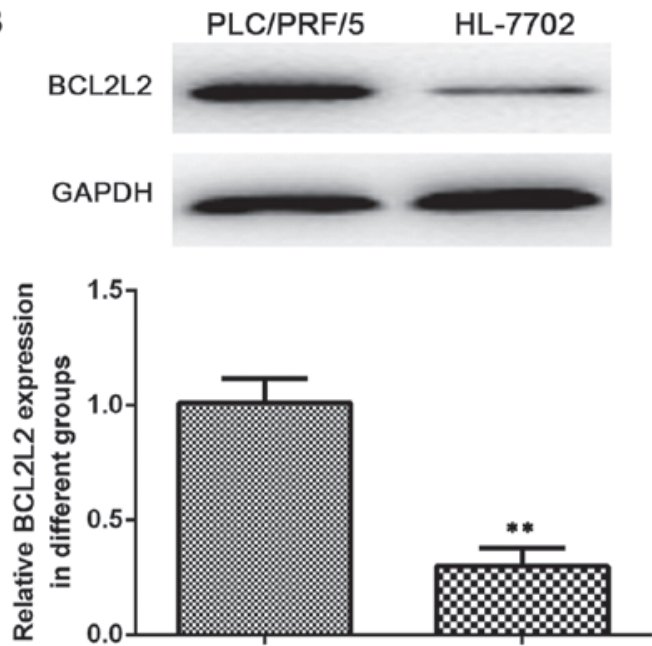

PLC/PRF/5

HL-7702

Figure 3. Determination of the TRIAP1 and BCL2L2 protein expression levels. (A) TRIAP1 expression in in hepatocellular carcinoma cells. (B) BCL2L2 expression in hepatocellular carcinoma cells. ${ }^{* *} \mathrm{P}<0.01$ vs. PLC/PRF/5 group. BCL2L2, B-cell lymphoma-2-like 2 protein; miR, microRNA; TRIAP1, TP53 regulated inhibitor of apoptosis 1 .

A

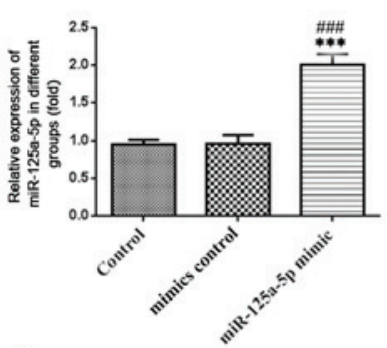

E

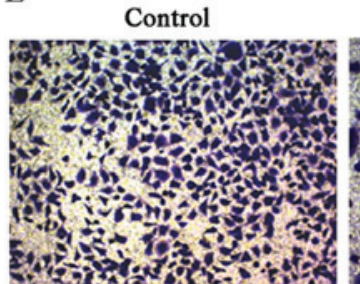

B

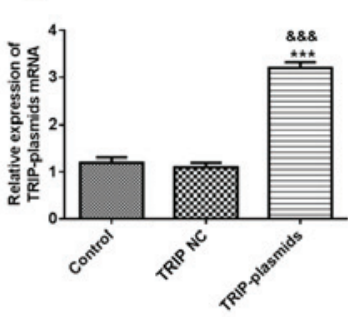

C

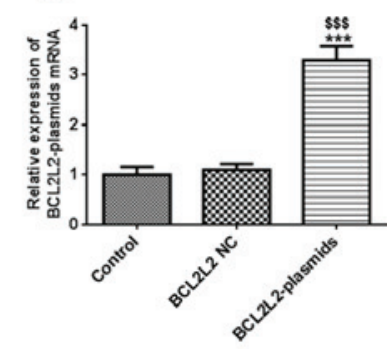

D

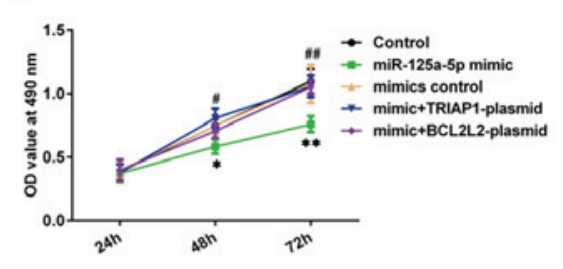

miR-125a-5p mimic

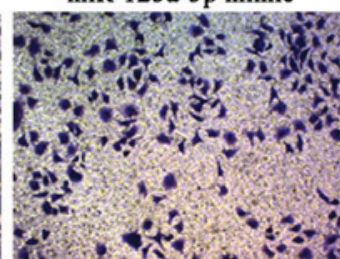

mimics control

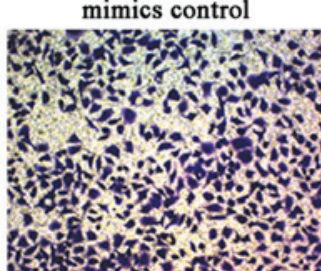

mimic+TRIAP1-plasmid

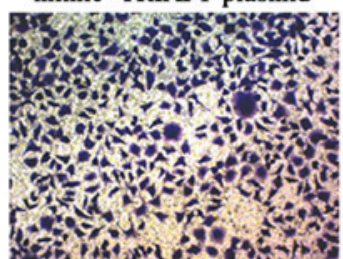

mimic+BCL2L2-plasmid

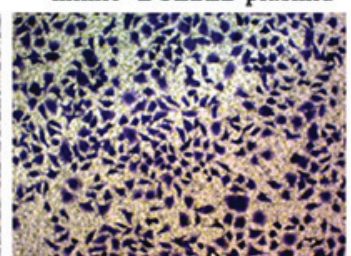

Figure 4. Effect of miR-125a-5p on cell viability and migration. (A) Reverse transcription-quantitative PCR confirmed the effective upregulation of miR-125a-5p upon transfection with miR-125a-5p mimic. (B) TRIAP1 was successfully overexpressed following transfection of the cells. (C) BCL2L2 was successfully overexpressed following transfection of the cells. (D) TRIAP1 and BCL2L2 overexpression increased the viability of the PLC/PRF/5 cells. (E) miR-125a-5p inhibits PLC/PRF/5 cell migration using the Transwell assay, which was reversed upon TRIP1 and BCL2L2 overexpression (magnification, $\mathrm{x} 200$ ). " $\mathrm{P}<0.05$, ${ }^{* *} \mathrm{P}<0.01$ and ${ }^{* * *} \mathrm{P}<0.001$ vs. control group; ${ }^{\# \mathrm{P}}<0.05$, ${ }^{\# \#} \mathrm{P}<0.01$ and ${ }^{\# \# \#} \mathrm{P}<0.001$ vs. mimics group; ${ }^{\& \& \&} \mathrm{P}<0.001$ vs. TRIP NC group; ${ }^{8 \$ \$} \mathrm{P}<0.001$ vs. BCL2L2 NC group. TRIP NC, TP53 regulated inhibitor of apoptosis 1 negative group; BCL2L2 NC, B-cell lymphoma-2-like 2 protein negative group; miR, microRNA.

line, HL-7702 were analyzed to determine the protein and mRNA expression levels of TRIAP1 (Fig. 3A) and BCL2L2 (Fig. 3B) using western blot assay and RT-qPCR respectively. These experiments demonstrated that the protein expression levels of TRIAP1 and BCL2L2 were markedly higher in the PLC/PRF/5 cells compared with the HL-7702 cells (Fig. 3A and B).

miR-125a-5p inhibits PLC/PRF/5 cell viability and migration. RT-qPCR analysis determined the upregulation of miR-125a-5p in PLC/PRF/5 cells, miR-125a-5p was overexpressed using miR-125a-5p mimic. After $48 \mathrm{~h}$ of cell transfection, the effective upregulation of the mRNA levels of miR-125a-5p as compared with the non-transfected and control-transfected cells was confirmed as observed in Fig. 4A. As shown in Fig. 4B and C, compared with the control group, the TRIAP1 was successfully overexpressed in TRIAP1 plasmids and BCL2L2 expression was up-regulated in BCL2L2 plasmids. To determine the effect of miR-125a-5p on hepatocellular carcinoma cell viability, MTT assay was performed. The results obtained revealed that the miR-125a-5p notably reduced the cell viability, but the inhibition was cancelled out upon TRIAP1 or BCL2L2 overexpression (Fig. 4D). Similarly, Transwell assay revealed that transfection with miR-125a-5p mimic markedly reduced the migration capacity of the PLC/PRF/5 cells compared with transfection with the control, 

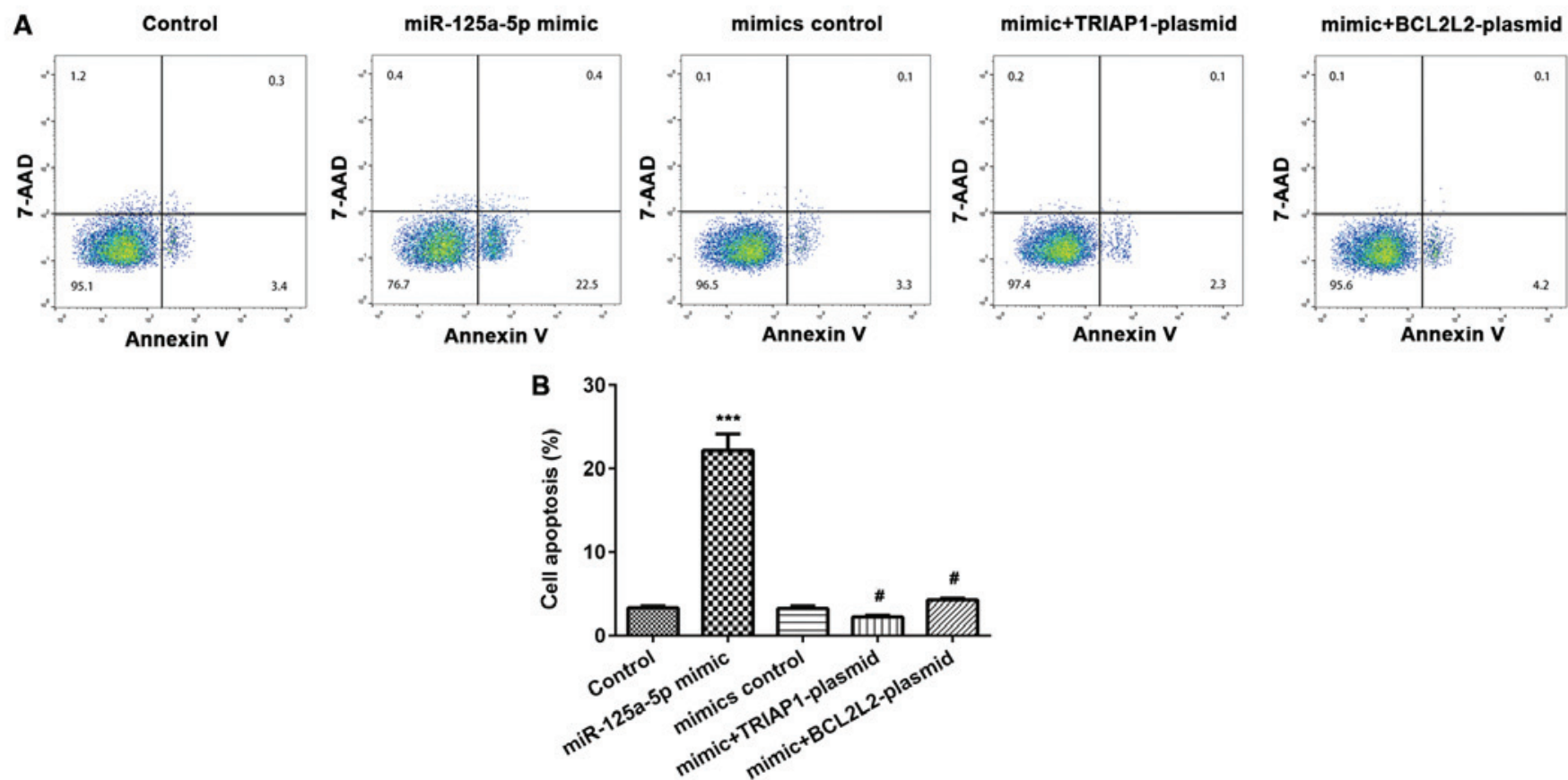

Figure 5. Apoptotic rates of all the groups. (A) Transfection of the cells with miR-125a-5p was shown to promote the apoptosis rate, whereas the apoptotic rates were reduced by TRIAP1 or BCL2L2 overexpression. (B) Histograms showing that miR-125a-5p mimic promoted the PLC/PRF/5 cell apoptosis rate. ${ }^{* * *} \mathrm{P}<0.001$ vs. control group; ${ }^{\text {"P}} \mathrm{P}<0.05$ vs. mimics control group. BCL2L2, B-cell lymphoma-2-like 2 protein; TRIAP1; miR, microRNA; TP53-regulated inhibitor of apoptosis 1.
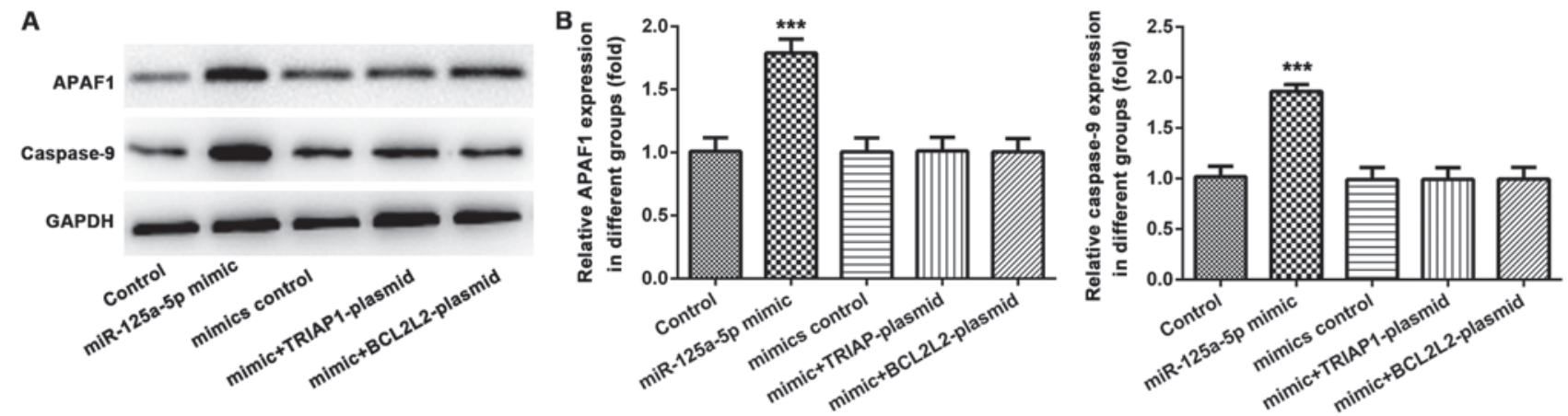

Figure 6. Expression of the apoptosis-associated proteins, caspase-9 and APAF1. (A) Transfection of miR-125a-5p upregulated caspase-9 and APAF1, whereas TR1AP1 or BCL2L2 overexpression inhibits the upregulation of caspase-9 and APAF1 in PLC/PRF/5 cells. (B) Histograms demonstrating that transfection of the cells with miR-125a-5p improved the expression levels of the two apoptosis-associated proteins studied. ${ }^{* * *} \mathrm{P}<0.001$ vs. control group. APAF1, apoptotic protease-activating factor 1; BCL2L2, B-cell lymphoma-2-like 2 protein; miR, microRNA; TRIAP1, TP53-regulated inhibitor of apoptosis 1.

but this effect was reversed when TRIAP1 or BCL2L2 were overexpressed (Fig. 4E).

miR-125a-5p induces PLC/PRF/5 cell apoptosis. Annexin VFITC/PI apoptosis detection kit was used to determine the extent of apoptosis of cells in the different groups. Compared with the control group, the rate of cell apoptosis was markedly enhanced in the miR-125a-5p mimic transfected PLC/PRF/5 cells (Fig. 5A). Furthermore, the effect of miR-125a-5p mimic on PLC/PRF/5 cell apoptosis was demonstrated to be reversed by TRIAP1 or BCL2L2 overexpression (Fig. 5B).

A previous study indicated that TRIAP1 inhibition could induce cell apoptosis by upregulating caspase-9 and apoptotic protease-activating factor 1 (APAF1) (17). Therefore, whether caspase-9 and APAF1 were involved in regulating PLC/PRF/5 cell apoptosis by miR-125a-5p was investigated using western blots. The results demonstrated that the protein levels of caspase-9 and APAF1 were markedly increased in miR-125a-5p transfected PLC/PRF/5 cells, and this effect was inhibited by either TRIAP1 or BCL2L2 overexpression (Fig. 6A and B).

\section{Discussion}

The present study demonstrated that miR-125a-5p was downregulated in the human hepatocellular carcinoma cell lines, PLC/PRF/5 and MHCC97L, but this effect was more noteworthy in PLC/PRF/5 cells. Further studies indicated that miR-125a-5p inhibited PLC/PRF/5 cell proliferation and invasion and induced cell apoptosis by targeting TRIAP1 and BCL2L2. Therefore, the findings presented in the present study suggested that miR-125a-5p may be a novel and promising therapeutic target for treatment of hepatocellular carcinoma. 
Hepatocellular carcinoma is the third leading cause of cancer- associated mortality in the world (18). Although the treatment methods have been improved in recent years, the recurrence and mortality rates associated with hepatocellular carcinoma continue to increase, and the prognosis of patients is extremely poor (19). The predominant causes of high mortality in hepatocellular carcinoma are the metastasis and recurrence of hepatocellular carcinoma. Since the exact mechanism remains unclear, there continues to be a lack of effective means of prevention and treatment. Therefore, it is required to find novel and effective targets for the diagnosis and treatment of hepatocellular carcinoma.

miRNAs, a class of non-coding small RNAs, have been revealed to serve a similar role to proto-oncogenes or tumor suppressor genes in the development and progression of various types of malignancies $(20,21)$. Increasing evidence has demonstrated that the abnormal expression of miRNAs is involved in the occurrence and development of hepatocellular carcinoma $(22,23)$. miR-125a-5p, an extensively studied miRNA in tumors, has been identified to serve an inhibitor role in hepatitis B virus-related hepatocellular carcinoma (15). Jin et al (24) suggested that miR-125a-5p is associated with the clinical stages of head and neck squamous cell carcinoma, and the expression levels in low tumor stages (stages I and II) were higher compared with that in the high stages (stages III and IV). Furthermore, miR-125a-5p is a potential biomarker for acute ischemic stroke (25). However, the role and mechanism of miR-125a-5p in hepatocellular carcinoma remains largely unclear. Therefore, the present study was conducted.

Firstly, the expression levels of miR-125a-5p in human hepatocellular carcinoma cells were detected, and the results obtained confirmed that miR-125a-5p was downregulated in the human hepatocellular carcinoma cell line, PLC/PRF/5. Subsequently, in order to explore the role of miR-125a-5p in hepatocellular carcinoma, the potential targets of miR-125a-5p were predicted using the TargetScan database and were confirmed by dual luciferase reporter assay. These findings suggested that TRIAP1 and BCL2L2 were the targets of miR-125a-5p. Subsequently, the upregulation of TRIAP1 and BCL2L2 was investigated in PLC/PRF/5 cells. Further analysis indicated that miR-125a-5p inhibited PLC/PRF/5 cell proliferation and migration. As TRIAP1 and BCL2L2 are both apoptosis inhibitory factors $(17,26,27)$, whether miR-125a-5p exerted an effect on cell apoptosis was subsequently investigated. As expected, it was shown that miR-125a-5p overexpression markedly induced PLC/PRF/5 cell apoptosis. TRIAP1 inhibition has been shown to induce cell apoptosis by enhancing the expression of caspase-9 and APAF1 (17). Therefore, in the present study, whether caspase-9 and APAF1 were involved in miR-125a-5p regulated $\mathrm{PLC} / \mathrm{PRF} / 5$ cell apoptosis was investigated. The results of these experiments suggested that the protein levels of caspase- 9 and APAF1 were markedly increased in miR-125a-5p transfected PLC/PRF/5 cells, indicating the involvement of TRIAP1/caspase9/APAF1 in $\mathrm{miR}-125 \mathrm{a}-5 \mathrm{p}$ regulated $\mathrm{PLC} / \mathrm{PRF} / 5$ cell apoptosis. Furthermore, an important finding of the present study was that all the effects of miR-125a-5p on PLC/PRF/5 cells were eliminated upon either TRIAP1 or BCL2L2 overexpression.

In conclusion, to the best of our knowledge, this is the first study to have revealed that miR-125a-5p may inhibit hepatocellular carcinoma cell growth and migration by directly targeting TRIAP1 and BCL2L2. Therefore, miR-125a-5p may be a novel and promising therapeutic target for the treatment of hepatocellular carcinoma.

\section{Acknowledgements}

Not applicable.

\section{Funding}

The present study was supported by Natural Science Fund Program of Xinjiang Uygur Autonomous Region (grant no. 2018D03011; China).

\section{Availability of data and materials}

The datasets used and/or analyzed during the current study are available from the corresponding author on reasonable request.

\section{Authors' contributions}

MM conceived and designed the experiments. MY,ML performed the experiments. ML analyzed the date. MM drafted the manuscript, MY and MM revised manuscript. The final version of the manuscript has been read and approved by all authors.

\section{Ethics approval and consent to participate}

Not applicable.

\section{Patient consent for publication}

Not applicable.

\section{Competing interests}

The authors declare that they have no competing interests.

\section{References}

1. Chiou JF, Tai CJ, Huang MT, Wei PL, Wang YH, An J, Wu CH, Liu TZ and Chang YJ: Glucose-regulated protein 78 is a novel contributor to acquisition of resistance to sorafenib in hepatocellular carcinoma. Ann Surg Oncol 17: 603-612, 2010.

2. Mazzaferro V, Regalia E, Doci R, Andreola S, Pulvirenti A, Bozzetti F, Montalto F, Ammatuna M, Morabito A and Gennari L: Liver transplantation for the treatment of small hepatocellular carcinomas in patients with cirrhosis. N Engl J Med 334: 693-699, 1996.

3. Bruix J and Sherman M; American Association for the Study of Liver Diseases: Management of hepatocellular carcinoma: An update. Hepatology 53: 1020-1022, 2011.

4. Greten TF and Sangro B: Targets for immunotherapy of liver cancer. J Hepatol: Sep 18, 2017 (Epub ahead of print).

5. Anindo MI and Yaqinuddin A: Insights into the potential use of microRNAs as biomarker in cancer. Int J Surg 10: 443-449, 2012.

6. Chandrasekaran K, Karolina DS, Sepramaniam S, Armugam A, Wintour EM, Bertram JF and Jeyaseelan K: Role of microRNAs in kidney homeostasis and disease. Kidney Int 81: 617-627, 2012.

7. Petrovic N, Ergün S and Isenovic ER: Levels of MicroRNA heterogeneity in cancer biology. Mol Diagn Ther 21: 511-523, 2017.

8. Meng F, Henson R, Wehbe-Janek H, Ghoshal K, Jacob ST and Patel T: MicroRNA-21 regulates expression of the PTEN tumor suppressor gene in human hepatocellular cancer. Gastroenterology 133: 647-658, 2007. 
9. Alhoot MA, Wang SM and Sekaran SD: RNA interference mediated inhibition of dengue virus multiplication and entry in HepG2 cells. PLoS One 7: e34060, 2012.

10. Jin Y, Wang J, Han J, Luo D and Sun Z: MiR-122 inhibits epithelial-mesenchymal transition in hepatocellular carcinoma by targeting Snaill and Snail2 and suppressing WNT/ $\beta$-cadherin signaling pathway. Exp Cell Res 360: 210-217, 2017.

11. Zhong L, Sun S, Shi J, Cao F, Han X and Chen Z: MicroRNA-125a-5p plays a role as a tumor suppressor in lung carcinoma cells by directly targeting STAT3. Tumour Biol 39: $1010428317697579,2017$.

12. Zheng H, Wu J, Shi J, Lu C, Wang Y, Sun Q, Zhang G and Zhao G: miR-125a-5p upregulation suppresses the proliferation and induces the cell apoptosis of lung adenocarcinoma by targeting NEDD9. Oncol Rep 38: 1790-1796, 2017.

13. Natalia MA, Alejandro GT, Virginia TJ and Alvarez-Salas LM MARK1 is a novel target for miR-125a-5p: Implications for cell migration in cervical tumor cells. Microrna 7: 54-61, 2018.

14. Cao Y, Tan S, Tu Y, Zhang G, Liu Y, Li D, Xu S, Le Z, Xiong J, Zou W, et al: MicroRNA-125a-5p inhibits invasion and metastasis of gastric cancer cells by targeting BRMS1 expression. Oncol Lett 15: 5119-5130, 2018.

15. Li G, Zhang W, Gong L and Huang X: MicroRNA 125a-5p inhibits cell proliferation and induces apoptosis in hepatitis $\mathrm{B}$ virus-related hepatocellular carcinoma by downregulation of ErbB3. Oncol Res 27: 449-458, 2019.

16. Livak KJ and Schmittgen TD: Analysis of relative gene expression data using real-time quantitative PCR and the 2(-Delta Delta C(T)) method. Methods 25: 402-408, 2001

17. Fook-Alves VL, de Oliveira MB, Zanatta DB, Strauss BE and Colleoni GW: TP53 regulated inhibitor of apoptosis 1 (TRIAP1) stable silencing increases late apoptosis by upregulation of caspase 9 and APAF1 in RPMI8226 multiple myeloma cell line. Biochim Biophys Acta 1862: 1105-1110, 2016.

18. Forner A, Llovet JM and Bruix J: Hepatocellular carcinoma. Lancet 379: 1245-1255, 2012.

19. Shimada K, Sakamoto Y, Esaki M, Kosuge T, Morizane C, Ikeda M, Ueno H, Okusaka T, Arai Y and Takayasu K: Analysis of prognostic factors affecting survival after initial recurrence and treatment efficacy for recurrence in patients undergoing potentially curative hepatectomy for hepatocellular carcinoma. Ann Surg Oncol 14: 2337-2347, 2007.
20. Xiao J, Yu W, Hu K, Li M, Chen J and Li Z: miR-92a promotes tumor growth of osteosarcoma by targeting PTEN/AKT signaling pathway. Oncol Rep 37: 2513-2521, 2017.

21. Xie F, Yuan Y, Xie L, Ran P, Xiang X, Huang Q, Qi G, Guo X, Xiao $C$ and Zheng S: miRNA-320a inhibits tumor proliferation and invasion by targeting c-Myc in human hepatocellular carcinoma. Onco Targets Ther 10: 885-894, 2017.

22. Zhu HR, Huang RZ, Yu XN, Shi X, Bilegsaikhan E, Guo HY, Song GQ, Weng SQ, Dong L, Janssen HLA, et al: Microarray expression profiling of microRNAs reveals potential biomarkers for hepatocellular carcinoma. Tohoku J Exp Med 245: 89-98, 2018.

23. Yang T, Thakur A, Chen T, Yang L, Lei G, Liang Y, Zhang S, Ren $\mathrm{H}$ and Chen M: MicroRNA-15a induces cell apoptosis and inhibits metastasis by targeting BCL2L2 in non-small cell lung cancer. Tumour Biol 36: 4357-4365, 2015.

24. Jin S, Liu MD, Wu H, Pang P, Wang S, Li ZN, Sun CF and Liu FY: Overexpression of hsa-miR-125a-5p enhances proliferation, migration and invasion of head and neck squamous cell carcinoma cell lines by upregulating $\mathrm{C}-\mathrm{C}$ chemokine receptor type 7. Oncol Lett 15: 9703-9710, 2018.

25. Li Y, Tang X, He Q, Yang X, Ren X, Wen X, Zhang J, Wang Y, Liu N and Ma J: Overexpression of mitochondria mediator gene TRIAP1 by miR-320b loss is associated with progression in nasopharyngeal carcinoma. PLoS Genet 12: e1006183, 2016.

26. Tiedt S, Prestel M, Malik R, Schieferdecker N, Duering M, Kautzky V, Stoycheva I, Böck J, Northoff BH, Klein M, et al: RNA-Seq identifies circulating miR-125a-5p, miR-125b-5p, and miR-143-3p as potential biomarkers for acute ischemic stroke. Circ Res 121: 970-980, 2017.

27. Wen Y, Han J, Chen J, Dong J, Xia Y, Liu J, Jiang Y, Dai J, Lu J, Jin G, et al: Plasma miRNAs as early biomarkers for detecting hepatocellular carcinoma. Int J Cancer 137: 1679-1690, 2015.

(c) (i) (9) This work is licensed under a Creative Commons CY Attribution-NonCommercial-NoDerivatives 4.0 International (CC BY-NC-ND 4.0) License. 